\title{
Temperature effects on the static and dynamic properties of liquid water inside nanotubes
}

\author{
J. Martí \\ Departament de Física i Enginyeria Nuclear, Universitat Politècnica de Catalunya, B5 Campus Nord, 08034 Barcelona, Catalonia, Spain \\ M. C. Gordillo \\ Departamento de Ciencias Ambientales, Facultad de Ciencias Experimentales, Universidad Pablo de Olavide. Carretera de Utrera Km 1, \\ 41013 Sevilla, Spain \\ and Departament de Física i Enginyeria Nuclear, Universitat Politècnica de Catalunya, B5 Campus Nord, 08034 Barcelona, \\ Catalonia, Spain \\ (Received 26 February 2001; published 18 July 2001)
}

\begin{abstract}
We report a molecular dynamics simulation study of the behavior of liquid water adsorbed in carbon nanotubes under different thermodynamic conditions. A flexible simple point charged potential has been employed to model internal and intermolecular water interactions. Water-carbon forces are modeled with a Lennard-Jones-type potential. We have studied three types of tubes with effective radii ranging from 4.1 to 6.8 $\AA$ and three temperatures, from 298 to $500 \mathrm{~K}$ for a fixed density of $1 \mathrm{~g} / \mathrm{cm}^{3}$. Structure of each thermodynamic state is analyzed through the characterization of the hydrogen-bond network. Time-dependent properties such as the diffusive behavior and molecular vibrational spectra are also considered. We observe the gradual destruction of the hydrogen-bond network together with faster diffusive regimes as temperature increases. A vibrational mode absent in bulk unconstrained water appears in the power spectra obtained from hydrogen velocity autocorrelation functions for all thermodynamic states. That frequency mode should be attributed to confinement effects.
\end{abstract}

DOI: 10.1103/PhysRevE.64.021504

PACS number(s): 61.25.-f, 31.15.Qg, 73.20.Mf

\section{INTRODUCTION}

Carbon nanotubes (CN's) are quasi-one-dimensional hollow structures that can be imagined as the result of rolling up one or several graphite sheets over themselves. These new materials sport a variety of novel electronic, structural and vibrational properties, among them the possibility of adsorbing substances in a quasi-one dimensional environment $[1,2]$. If the chosen substance is water, the interest is twofold: not only the general aim of understanding the behavior of adsorbates under the confinement of the CN's, but also the possibility of extrapolating the conclusions obtained to similar interesting physical situations, such as water adsorbed in biological or geological systems.

Some work has been done concerning liquid water confined in silica pores [3-5], but the confinement of water in nanotubes is tighter, since there are much narrower than the typical silica pores. We have recently performed several molecular dynamics (MD) calculations of liquid water confined in CN's at the room conditions of $298 \mathrm{~K}$ and $1 \mathrm{~g} / \mathrm{cm}^{3}$ : we analyzed the hydrogen-bond network structure [6] and the vibrational and rotational spectra [7]. Later we have also studied the influence of atomic mass on the microscopic dynamics of water using isotopic substitutions [8].

In this paper we present an analysis of both structural and time-dependent properties of liquid water as a function of the temperature by means of MD simulations. Preliminary studies of such properties as a function of the density have shown few changes from the qualitative point of view for variations up to the $25 \%$ of the density. The main objectives of this work are the study of the changes produced by the increasing temperature on the hydrogen-bond network and the influence of those changes on the dynamic properties. We will also be concerned with the analysis of the diffusive behavior of the confined liquid water, and finally with the computation of microscopic spectra for all systems. Special attention will be devoted to the evolution of the anomalous frequency bands absent in bulk room temperature water when the temperature changes. The potential models and computational tools will be described in Sec. II and we will show structure and hydrogen-bond populations for all states in Sec. III. Selfdiffusion coefficients along the tube axis will be reported in Sec. IV, while the computed spectral densities in the full infrared spectra regions will be described in Sec. V. Section VI is devoted to conclusions.

\section{COMPUTATIONAL DETAILS}

We have performed a series of MD simulations of $\mathrm{H}_{2} \mathrm{O}$ confined in CN's with internal radii of 4.10, 5.45, and 6.80 $\AA$, which correspond to the $(6,6),(8,8)$, and $(10,10)$ tubes in the standard nomenclature [9], at three different temperatures and the density of $1 \mathrm{~g} / \mathrm{cm}^{3}$. The details of the simulated systems are reported in Table I. We have modeled waterwater interactions by means of a flexible version [10] of the simple point charged (SPC) potential of Berendsen et al. [11]. SPC is one of the most used potentials in the literature and it consists in a Lennard-Jones type term to model shortrange interactions plus a Coulombic part to account for long ranged forces. The model employed in this work includes intramolecular interactions that account for the internal vibrational modes of water. This flexible potential has been previously checked through the comparison with experimental infrared and Raman spectra for systems at several thermodynamic conditions [12]. Other water samples such as heavy water steam and water at supercritical conditions have also been studied with this model [13-15]. Water-tube interactions have been accounted for through Lennard-Jones type 
TABLE I. Simulated systems. All simulations have been performed at a density of $1.00 \mathrm{~g} / \mathrm{cm}^{3}$.

\begin{tabular}{ccccc}
\hline \hline Simulation & $\begin{array}{c}\text { Tube radius } \\
(\AA)\end{array}$ & Tube class & $\begin{array}{c}\text { Number } \\
\text { of waters }\end{array}$ & Temperature (K) \\
\hline 1 & 4.10 & $(6,6)$ & 14 & 298 \\
2 & 5.45 & $(8,8)$ & 56 & 298 \\
3 & 6.80 & $(10,10)$ & 126 & 298 \\
4 & 4.10 & $(6,6)$ & 14 & 400 \\
5 & 5.45 & $(8,8)$ & 56 & 400 \\
6 & 6.80 & $(10,10)$ & 126 & 400 \\
7 & 4.10 & $(6,6)$ & 14 & 500 \\
8 & 5.45 & $(8,8)$ & 56 & 500 \\
9 & 6.80 & $(10,10)$ & 126 & 500 \\
\hline \hline
\end{tabular}

potentials [16]. The shapes and parameters of the carbonatom interactions are reported elsewhere [6,7].

Standard periodic boundary conditions have been considered and the Ewald rule has been employed to sum up the long-ranged Coulomb interactions. We also used a leap-frog Verlet integration algorithm with coupling to a Berendsen thermostat [17] and a time step of 0.5 fs for all simulations. In addition, translational and internal degrees of freedom have been independently equilibrated [18] in order to quickly achieve the desired temperatures. We performed MD runs longer than $0.5 \mathrm{~ns}$ to collect statistics after equilibration runs longer than $0.1 \mathrm{~ns}$.

\section{STRUCTURE AND HYDROGEN-BOND NETWORK}

The analysis of the structure of liquid water confined in CN's has been performed through the computation of hydrogen-bond (HB) populations and atomic density profiles. We have employed a geometrical definition of hydrogen bonding [6] that has been also assumed in many molecular dynamics calculations $[14,19,20]$. The HB percentages for our constrained water systems are reported in Table II for molecules involved in 0-5 HB's and compared with the val- ues corresponding to bulk systems for each temperature. We can distinguish several general trends concerning the average HB number per molecule $\left\langle n_{H B}\right\rangle$. One, $\left\langle n_{H B}\right\rangle$ in the constrained water systems is lower than the one in bulk systems for all temperatures. Two, $\left\langle n_{H B}\right\rangle$ tends to decrease as temperature increases for each nanotube class and three, $\left\langle n_{H B}\right\rangle$ grows with increasing tube radius at each particular temperature. Besides, when we raise the temperature, there is a great increase in the percentage of molecules with zero or one hydrogen-bond, and a related decrease in the number of multiple bound molecules. At 298 and $400 \mathrm{~K}$, the maxima in the hydrogen bond distributions for all tubes are located at lower number of HB's $\left(n_{H B}=3\right.$ at room temperature versus $n_{H B}$ $=4$ for bulk and $n_{H B}=2$ versus $n_{H B}=3$ in the $400 \mathrm{~K}$ case). However, at $500 \mathrm{~K}$, the peaks are located at $n_{H B}=2$, both for bulk and constrained water. This probably indicates that the destruction of bonds induced by the presence of the nanotube starts to be balanced by the decreasing produced by temperature itself. That would explain why the percentages of water molecules forming $0-5 \mathrm{HB}$ are very similar for the wider tubes $(8,8)$ and $(10,10)$ at 298 and $400 \mathrm{~K}$, but not for $500 \mathrm{~K}$. The case of the $(6,6)$ tube is different: its HB distribution shows the effects of the reduced space available to the water molecules. The fact that at $500 \mathrm{~K},\left\langle n_{H B}\right\rangle<2$ in all cases, indicates that we have long chains of hydrogen bonds.

To complete the structural study, we show the average number of hydrogen bonds as a function of the distance to the $z$ axis in Fig. 1. We observe that the shape of the distributions is similar for each of the three tubes considered, differing only in its height, as corresponds to cases with different HB populations. Figures 2 and 3 show atomic density profiles (probability of finding an atom at a fixed distance from the longitudinal axis) for different temperatures. In line with what we mentioned above, we obtain that at all temperatures the oxygen and hydrogen distributions are very similar, independent of their respective hydrogen-bond distributions. This could be attributed to confinement effects, which are really dominant in these systems, independent of the hydrophobic or hydrophilic type of the interaction. This

TABLE II. Averaged percentages of H-bond populations. Error estimates are reported in parentheses. Bulk water data are taken from Refs. [14,20]. At $298 \mathrm{~K}$, there are the $0.5 \%$ of waters forming $6 \mathrm{HB}$.

\begin{tabular}{lccccccc}
\hline \hline Simulation & $N_{0}$ & $N_{1}$ & $N_{2}$ & $N_{3}$ & $N_{4}$ & $N_{5}$ & $\langle N\rangle$ \\
\hline 1 & $1.4(0.1)$ & $19.5(0.2)$ & $79.1(0.2)$ & 0 & 0 & 0 & 1.8 \\
2 & $0.3(0.1)$ & $4.3(0.2)$ & $30.4(0.4)$ & $46.4(0.4)$ & $18.6(0.5)$ & 0 & 2.8 \\
3 & $0.2(0.1)$ & $3.8(0.3)$ & $26.9(0.6)$ & $45.6(0.6)$ & $22.4(0.5)$ & $1.1(0.2)$ & 2.9 \\
Bulk (298 K) & $<0.1$ & 0.3 & 4.8 & 26.0 & 58.2 & 10.2 & 3.7 \\
\hline 4 & $13.1(0.1)$ & $21.6(0.2)$ & $65.3(0.2)$ & 0 & 0 & 0 & 1.5 \\
5 & $3.2(0.2)$ & $19.5(0.5)$ & $48.2(0.6)$ & $25.5(0.6)$ & $3.6(0.2)$ & 0 & 2.1 \\
6 & $2.1(0.3)$ & $15.3(0.7)$ & $41.8(0.8)$ & $31.7(0.8)$ & $8.6(0.5)$ & $0.4(0.1)$ & 2.3 \\
Bulk (403 K) & 0.5 & 5.6 & 23.0 & 39.4 & 28.4 & 3.1 & 3.0 \\
\hline 7 & $18.0(0.2)$ & $25.0(0.2)$ & $57.0(0.3)$ & 0 & 0 & 0 & 1.4 \\
8 & $10.4(0.5)$ & $32.2(0.8)$ & $41.5(0.8)$ & $14.4(0.6)$ & $1.5(0.2)$ & 0 & 1.6 \\
9 & $6.1(0.5)$ & $26.2(0.8)$ & $41.8(0.8)$ & $21.4(0.7)$ & $4.3(0.4)$ & $0.2(0.1)$ & 1.9 \\
Bulk (523 K) & 3.2 & 17.6 & 36.0 & 30.8 & 11.4 & 1.0 & 2.3 \\
\hline \hline
\end{tabular}




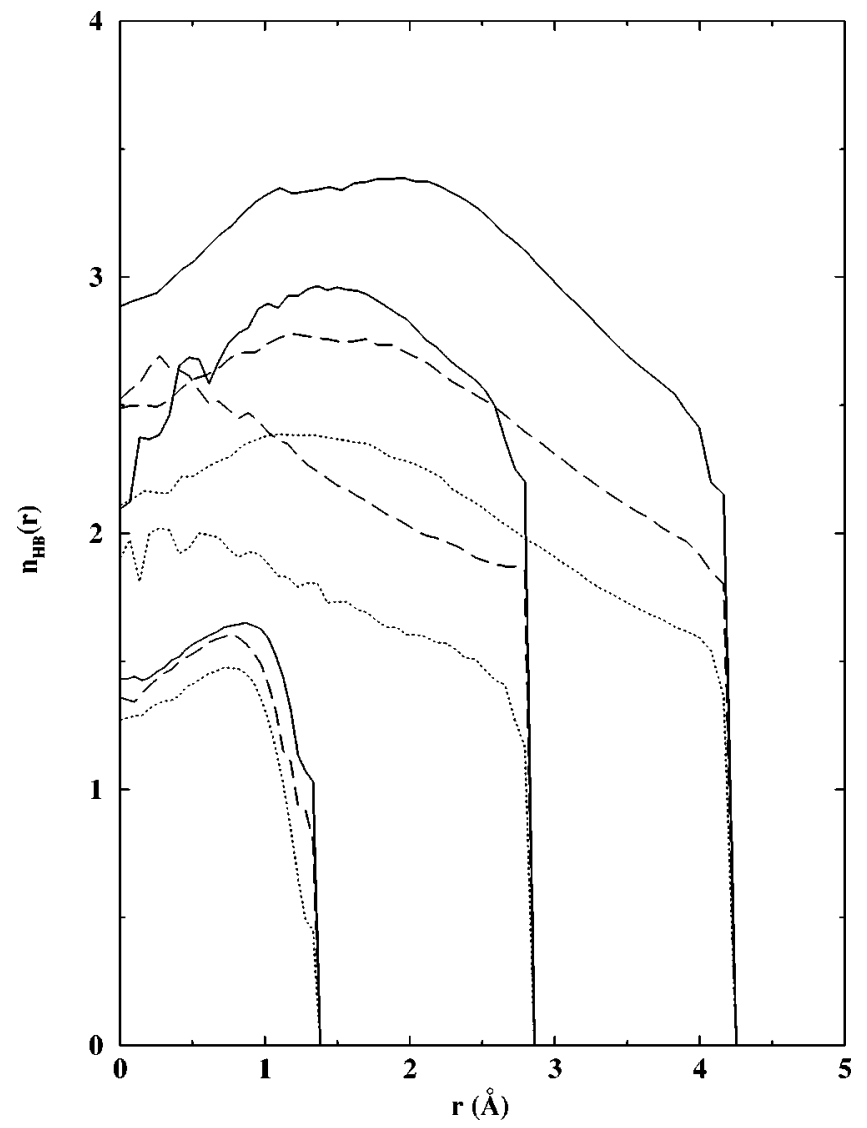

FIG. 1. Averaged number of H bonds for water in CN's. $298 \mathrm{~K}$ (continuous lines), $400 \mathrm{~K}$ (dashed lines), $500 \mathrm{~K}$ (dotted lines).

has been already observed for adsorbed water in the proximity of different substrates [21-26].

\section{SELF-DIFFUSION COEFFICIENTS}

In this work we have employed the velocity autocorrelation functions of the oxygen atoms, $C(t)$ 's, together with their $z$ components $C_{z}(t)$ 's, in order to compute the water self-diffusion coefficients $D$ 's. These are the total (isotropic) $D, z$ axis, $D_{z}$, and the one for the $x y$ plane, $D_{x y}$. The relationship between both quantities is given by

$$
D=\frac{1}{3} \int_{0}^{\infty} d t C(t),
$$

and the results are reported in Table III. First of all, we see that our flexible potential model produces a good qualitative agreement with the two experimental data available. However, it should be pointed out that only experimental bulk data are reported. We also observe that diffusion along $z$ axis is faster than in the $x y$ plane for all tubes, this being specially important for the $(6,6)$ one. The lack of space is responsible for the great differences between $D_{x y}$ 's in the $(6,6),(8,8)$, and $(10,10)$ tubes. We observe also a great increase in $D_{z}$ in going from room temperature to $400 \mathrm{~K}$. This is probably

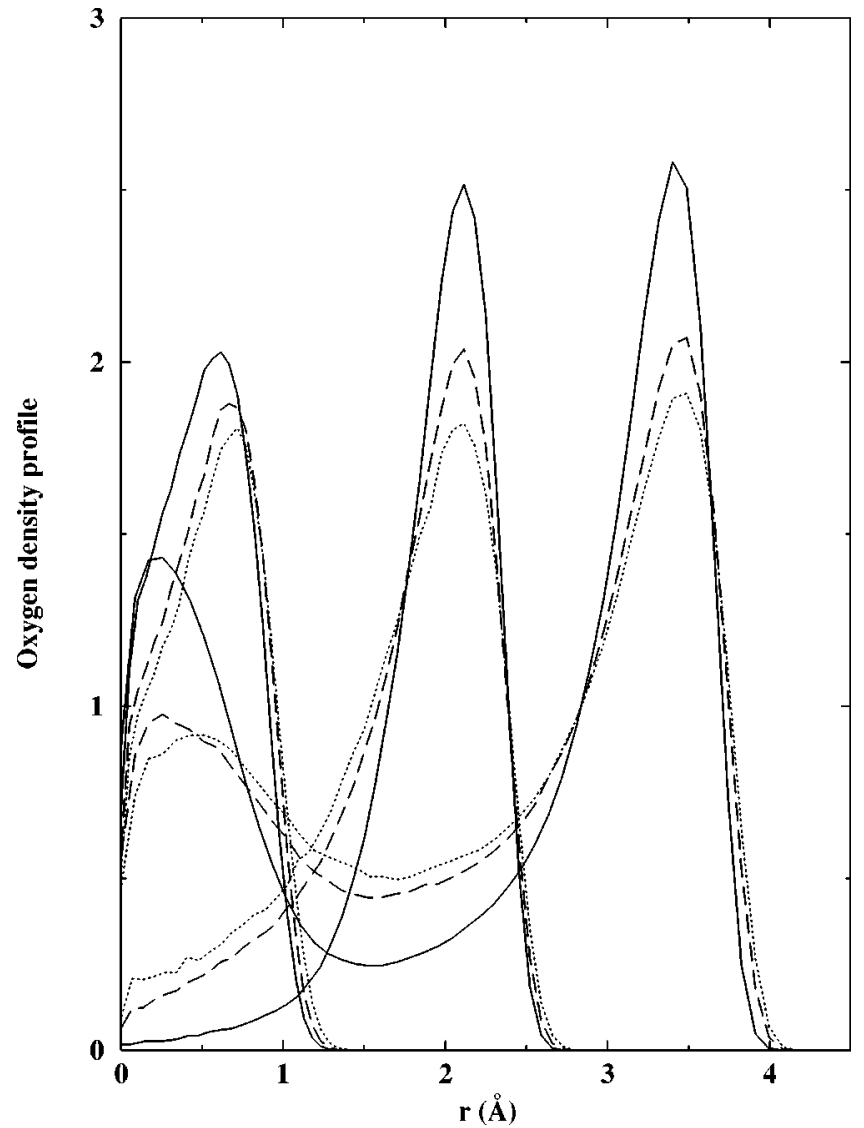

FIG. 2. Oxygen density profiles (in $\mathrm{g} / \mathrm{cm}^{3}$ ) for CN. $298 \mathrm{~K}$ (continuous lines), $400 \mathrm{~K}$ (dashed lines), and $500 \mathrm{~K}$ (dotted lines).

correlated with the destruction of hydrogen bonds, since the great reduction in $\left\langle n_{H B}\right\rangle$ produced between these two temperatures.

\section{VIBRATIONAL SPECTRA}

The calculation of spectral densities from microscopic correlation functions is one of the most useful ways to study microscopic dynamics [29], together with the experimental infrared and Raman spectra measurements. We have employed the hydrogen velocity autocorrelation functions $C_{H}(t)$ to compute the spectral densities $S_{H}(\omega)$. The procedure is limited to the obtention of the spectroscopic band maxima and works through the computation of the optical absorption line shape $I(\omega)$ by Fourier transforming the total dipole moment autocorrelation function. In this scheme, $I(\omega)$ can be directly related to $S_{H}(\omega)$. The details of the calculation are reported elsewhere $[8,12]$. The results are displayed in Figs. 4 and 5 and are complemented by the data in Table IV.

The hydrogen spectral densities $S_{H}(\omega)$ for liquid water at three temperatures are shown in Fig. 4. We compare $S_{H}(\omega)$ for water confined in CN's with the bulk water spectrum in the frequency range of $0-1000$ wave numbers. One can see that the spectral densities of the two wider tubes are very similar to each other at any temperature, while the $S_{H}(\omega)$ of the narrow $(6,6) \mathrm{CN}$ presents a very different shape. For all 


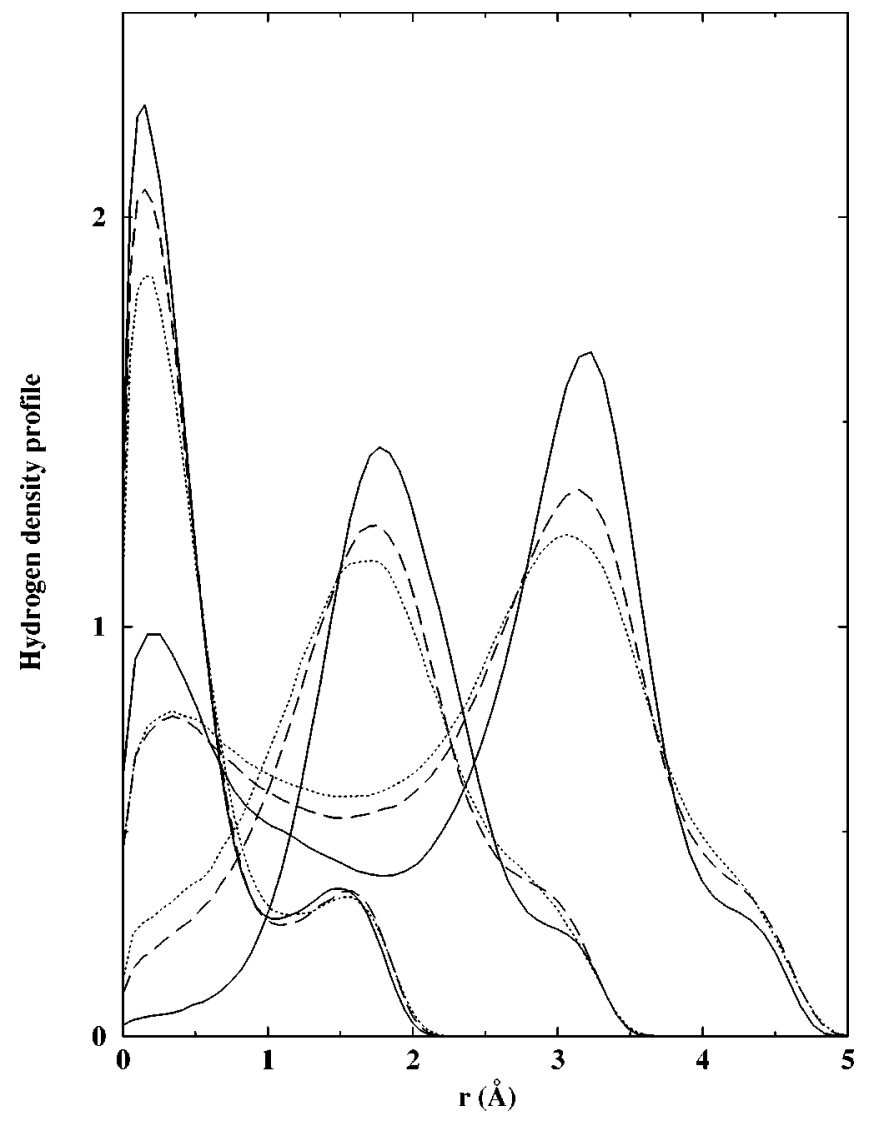

FIG. 3. Hydrogen density profiles (in $\mathrm{g} / \mathrm{cm}^{3}$ ) for $\mathrm{CN}$ at different temperatures. Full, dashed, and dotted lines have the same meaning as Fig. 2.

tubes and all temperatures, we can observe three different peaks: one at around $50 \mathrm{~cm}^{-1}$, related to the bending of O-O-O hydrogen-bonded units according to some authors [30], and to the "cage effect" according to others [20,31];

TABLE III. Oxygen and hydrogen self-diffusion coefficients along the tube axis. All values are given in $10^{-5} \mathrm{~cm}^{2} / \mathrm{s}$. Bulk water data are taken from Refs. [14,20].

\begin{tabular}{lccc}
\hline \hline Simulation & $D_{\text {total }}$ & $D_{\text {Z-axis }}$ & $D_{X Y \text {-plane }}$ \\
\hline 1 & 2.5 & 4.3 & 1.6 \\
2 & 3.2 & 3.5 & 3.0 \\
3 & 3.1 & 3.8 & 2.7 \\
Bulk (298 K) & 2.6 & & \\
Experimental (298 K) [27] & 2.3 & & \\
\hline 4 & 10.6 & 29.2 & 1.3 \\
5 & 11.0 & 16.9 & 8.0 \\
6 & 11.2 & 15.1 & 9.2 \\
Bulk (403 K) & 9.9 & & \\
\hline 7 & 11.2 & 32.1 & 0.7 \\
8 & 15.9 & 29.6 & 9.0 \\
9 & 17.7 & 26.6 & 13.2 \\
Bulk (523 K) & 24.1 & & \\
Experimental (523 K) [28] & 28.0 & & \\
\hline \hline
\end{tabular}

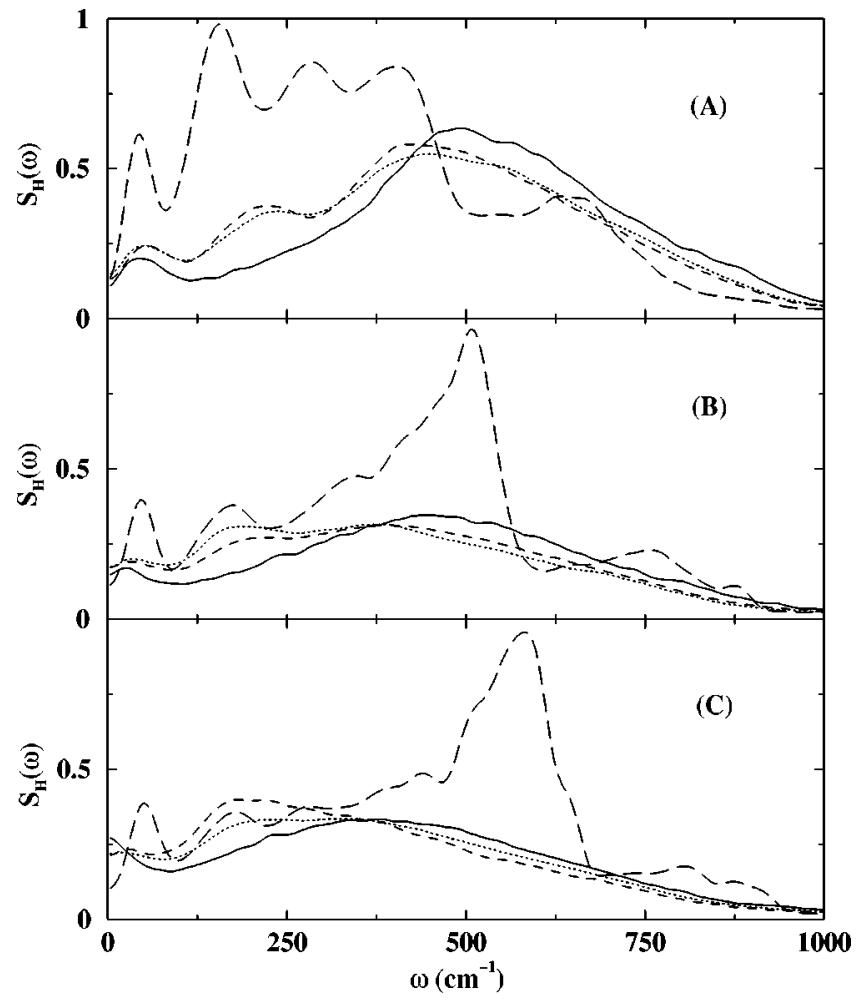

FIG. 4. Hydrogen spectral densities $S_{H}(\omega)$ (arbitrary units) for $\mathrm{CN}$ at different temperatures. Low frequency range (0-1000 $\mathrm{cm}^{-1}$ ). (A) $298 \mathrm{~K}$, (B) $400 \mathrm{~K}$, (C) $500 \mathrm{~K}$. Bulk is represented by full lines at ambient conditions, $(6,6) \mathrm{CN}$ are represented by longdashed lines, $(8,8) \mathrm{CN}$ are represented by dashed lines, and $(10,10)$ $\mathrm{CN}$ are represented by dotted lines. Bulk has been computed at 298, 403 , and $523 \mathrm{~K}$.

another at approximately $200 \mathrm{~cm}^{-1}$ related to the stretching of hydrogen bonds and a final one at about $400 \mathrm{~cm}^{-1}$. The first one is visible at frequencies above the corresponding bulk values. Since the breaking of the hydrogen bond network in $\mathrm{CN}$ is greater than in bulk we could suspect that, at least in nanotubes, the position of this band is related to the size of the "cage" available for each molecule: higher frequencies correspond to narrower tubes and smaller cages. However, this correspondence is not seen at room temperature, which suggests that the vibration of the entire hydrogen bond network also plays a role in the appearance of this band. The peak at $400 \mathrm{~cm}^{-1}$, related to the rotations of the water molecule, has a similar behavior in the wider tubes and in bulk, the only difference being its position, that depends on the diameter of the tube [7]. The $(6,6)$ tube presents a much more complicated behavior. Below $700 \mathrm{~cm}^{-1}$ we can observe four different peaks. The first two are related to the two bands already mentioned for bulk water (50 and $200 \mathrm{~cm}^{-1}$ ). Then, there are two maxima that we can tentatively assign to rotations; their frequencies are displayed in Table IV. When the temperature increases, these peaks shift forward enormously, one of them being in the vicinity of $600 \mathrm{~cm}^{-1}$. A band in this location has been observed in simulations of absorbed HDO (species resulting from the $\mathrm{H}_{2} \mathrm{O}-\mathrm{D}_{2} \mathrm{O}$ mixture) [8], and it could be attributed to an optical mode found in liquid bulk water [12,32]. 


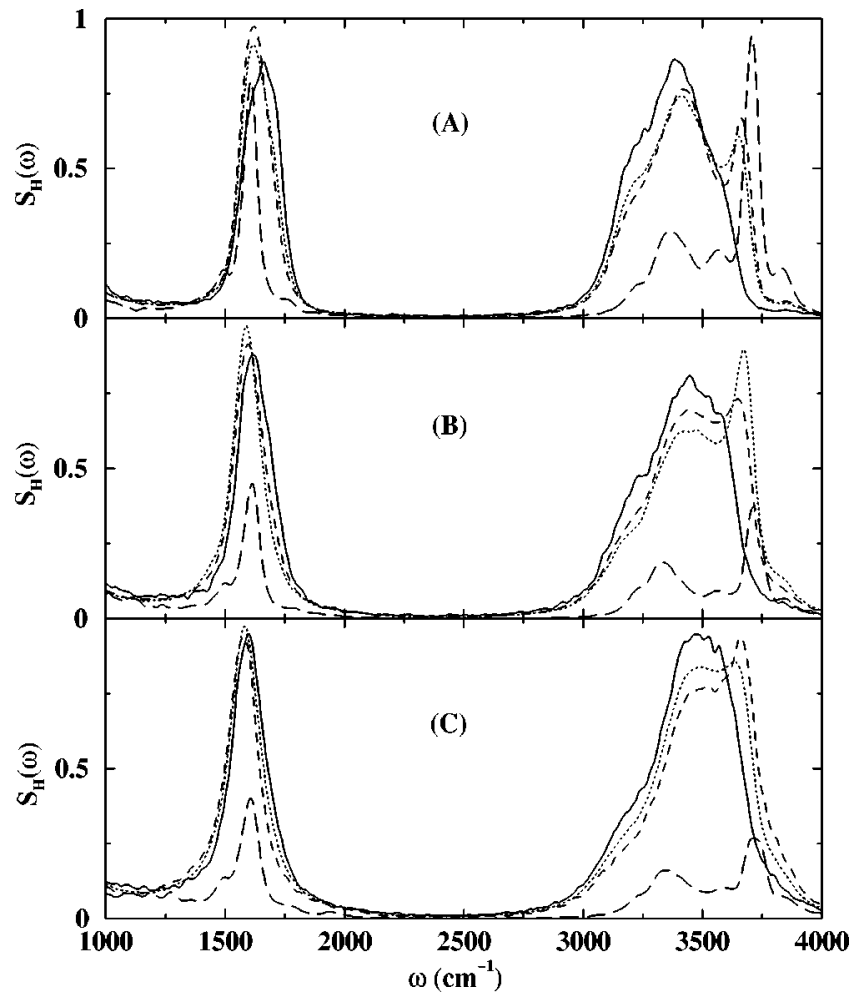

FIG. 5. Same as Fig. 4 in the high frequency range (1000-4000) wave numbers.

The high frequency domain between 1000 and 4000 wave numbers is displayed in Fig. 5. There we find the bending and stretching intramolecular vibrations. In all cases, the bending band is slightly backshifted (relative to the bulk value, at all temperatures) although we do not find significant differences for the three different tube classes. Important differences arise in the case of the stretching frequency, however: the band is splitted into two branches at all temperatures in such a way that the high frequency branch, which we call "vibrational" band, shifts forward proportionally to the inverse of tube radius. This has already been seen at room temperature [7] and we observe here that at higher temperatures the behavior is basically the same. In previous works $[7,8]$, we postulated that this splitting has to be associated with confinement effects and that it can be explained as the separation of the two stretching modes, the symmetric and the antisymmetric ones. The vibrational frequency has to be related with the antisymmetric stretch.

\section{CONCLUDING REMARKS}

We have performed a series of MD simulations of water adsorbed in CN's. Our interest has been the study of the microscopic structure and dynamics of liquid water as a function of the temperature as well as of the tube type. In order to analyze the intramolecular vibrations of water we have employed a flexible potential model previously tested. The hydrogen-bond network of constrained water has been revealed to be weaker than the one of bulk water at all simu-
TABLE IV. Frequency maxima (in $\mathrm{cm}^{-1}$ ) for water confined in $\mathrm{CN}$ computed from hydrogen spectral densities $S_{H}(\omega)$. The frequencies $\omega_{1}$ and $\omega_{2}$ are the two typical intermolecular vibrations of liquid water. The estimated statistical uncertainty is around $3 \mathrm{~cm}^{-1}$ for all values. Bulk water data are taken from Refs. [14,20].

\begin{tabular}{lcccccc}
\hline \hline Simulation & $\omega_{1}$ & $\omega_{2}$ & $\omega_{\text {Rotation }}$ & $\omega_{\text {Bending }}$ & $\omega_{\text {Stretching }}$ & $\omega_{\text {Vibration }}$ \\
\hline 1 & 45 & 155 & $285-400$ & 1606 & 3363 & 3707 \\
2 & 54 & 223 & 420 & 1620 & 3420 & 3662 \\
3 & 57 & 243 & 448 & 1615 & 3412 & 3657 \\
Bulk (298 K) & 50 & 200 & 505 & 1650 & 3385 & \\
\hline 4 & 45 & 175 & $345-508$ & 1612 & 3330 & 3710 \\
5 & 35 & 190 & 377 & 1590 & 3450 & 3673 \\
6 & 32 & 220 & 387 & 1595 & 3445 & 3650 \\
Bulk (403 K) & 20 & 190 & 455 & 1620 & 3460 & \\
\hline 7 & 50 & 178 & $440-580$ & 1605 & 3350 & 3714 \\
8 & 27 & 200 & 350 & 1580 & 3485 & 3660 \\
9 & 23 & 225 & 335 & 1580 & 3495 & 3630 \\
Bulk (523 K) & 0 & 180 & 370 & 1590 & 3480 & \\
\hline \hline
\end{tabular}

lated temperatures. This fact has been observed through the computation of hydrogen-bond populations and atomic density profiles. We observed that narrow tubes do not allow complex H-bonded structures and, in many cases, the adsorbed water molecules inside are forming long linear chains. Diffusive motion has been studied from isotropic self-diffusion coefficients as well as those computed along the longitudinal tube axis. We have found that diffusion in the $z$ direction is clearly faster than the bulk value in all cases.

The calculation of the spectral densities of oxygen and hydrogen atoms has reported a set of remarkable results: first, the presence of the tube walls produce shifts (referred to the bulk values) in most of the spectral bands. Some of these frequency shifts can be related to the tube radii and are also produced in the same tube classes at different temperatures. One relevant fact is the detection of a high vibrational frequency in the stretching spectral region of all constrained water samples which is absent in the bulk. This frequency mode can be explained as the splitting of the stretching symmetric and antisymmetric components and it has been detected at each temperature studied. The low frequency band around $50 \mathrm{~cm}^{-1}$ in liquid bulk water is also present in the confined systems.

\section{ACKNOWLEDGMENTS}

Project No.1999SGR-00146 of the Direcció General de Recerca de la Generalitat de Catalunya, Project Nos. BFM2000-0596-C03-02 and PB98-0922 of the Ministerio de Educación y Cultura of Spain and PR99-05 of the Universitat Politècnica de Catalunya are acknowledged for financial support. M.C.G. also thanks the Spanish Ministry of Education and Culture for a postgraduate contract. 
[1] M.S. Dresselhaus, G. Dresselhaus, P.C. Eklund, and R. Saito, Phys. World 11, 33 (1998).

[2] R. Saito, G. Dresselhaus, and M.S. Dresselhaus, Physical Properties of Carbon Nanotubes (Imperial College Press, London, 1998).

[3] F. Bruni, M.A. Ricci, and A.K. Soper, J. Chem. Phys. 109, 1478 (1998).

[4] A.K. Soper, F. Bruni, and M.A. Ricci, J. Chem. Phys. 109, 1486 (1998).

[5] C. Hartnig, W. Witschel, E. Spohr, P. Gallo, M.A. Ricci, and M. Rovere, J. Mol. Liq. 85, 127 (2000).

[6] M.C. Gordillo and J. Martí, Chem. Phys. Lett. 329, 341 (2000).

[7] J. Martí and M.C. Gordillo, Phys. Rev. B 63, 165430 (2001).

[8] J. Martí and M.C. Gordillo, J. Chem. Phys. 114, 10486 (2001).

[9] N. Hamada, S. Sawada, and A. Oshiyama, Phys. Rev. Lett. 68, 1579 (1992).

[10] J. Martí, J.A. Padró, and E. Guàrdia, J. Mol. Liq. 62, 17 (1994).

[11] H.J.C. Berendsen, J.P.M. Postma, W.F. van Gunsteren, and J. Hermans, in Intermolecular Forces, edited by B.Pullman (Reidel, Dordrecht, Holland, 1981).

[12] J. Martí, E. Guàrdia, and J.A. Padró, J. Chem. Phys. 101, 10883 (1994).

[13] J. Martí, J.A. Padró, and E. Guàrdia, Mol. Phys. 86, 263 (1995).

[14] J. Martí, J. Chem. Phys. 110, 6876 (1999).

[15] J. Martí, Phys. Rev. E 61, 449 (2000).
[16] G. Stan and M.W. Cole, Surf. Sci. 395, 280 (1997).

[17] H.J.C. Berendsen, J.P.M. Postma, W.F. van Gunsteren, A. DiNola, and J.R. Haak, J. Phys. Chem. 81, 3684 (1984).

[18] A. Wallqvist and O. Telemann, Mol. Phys. 74, 515 (1991).

[19] A. Luzar and D. Chandler, J. Chem. Phys. 98, 8160 (1993).

[20] J. Martí, J.A. Padró, and E. Guàrdia, J. Chem. Phys. 105, 639 (1996).

[21] C.Y. Lee, J.A. McCammon, and P.J. Rossky, J. Chem. Phys. 80, 4448 (1984).

[22] S.-B. Zhu and G.W. Robinson, J. Chem. Phys. 94, 1403 (1991).

[23] S.H. Lee and P.J. Rossky, J. Chem. Phys. 100, 3334 (1994).

[24] E. Spohr, J. Chem. Phys. 106, 388 (1997).

[25] J.X. Fang, W.H. Marlow, J.X. Lu, and R.R. Lucchese, J. Chem. Phys. 107, 5212 (1997).

[26] E. Spohr, C. Hartnig, P. Gallo and M. Rovere, J. Mol. Liq. 80, 165 (1999).

[27] K. Krynicki, C.D. Green, and D.W. Sawyer, Faraday Discuss. Chem. Soc. 66, 199 (1978).

[28] R. Hausser, G. Maier, and F. Noack, Z. Naturforsch. A 21A, 1410 (1966).

[29] J. Martí, J.A. Padró, and E. Guàrdia, Mol. Simul. 11, 336 (1993).

[30] G.E. Walrafen, in Water: A Comprehensive Treatise, edited by F. Franks (Plenum Press, New York, 1972).

[31] T. Nakayama, Phys. Rev. Lett. 80, 1244 (1998).

[32] H. Resat, F.O. Raineri, and H.L. Friedman, J. Chem. Phys. 97, 2618 (1992). 